University of Nebraska - Lincoln

DigitalCommons@University of Nebraska - Lincoln

4-15-1997

\title{
Magnetic hardening in FePt nanostructured films
}

J. Ping Liu

University of Nebraska-Lincoln, pliu@uta.edu

Yi Liu

University of Nebraska-Lincoln, yliu@unl.edu

C.P. Luo

University of Nebraska - Lincoln

Z.S. Shan

University of Nebraska - Lincoln

Follow this and additional works at: https://digitalcommons.unl.edu/physicssellmyer

Part of the Physics Commons

Liu, J. Ping; Liu, Yi; Luo, C.P.; and Shan, Z.S., "Magnetic hardening in FePt nanostructured films" (1997). David Sellmyer Publications. 82.

https://digitalcommons.unl.edu/physicssellmyer/82

This Article is brought to you for free and open access by the Research Papers in Physics and Astronomy at DigitalCommons@University of Nebraska - Lincoln. It has been accepted for inclusion in David Sellmyer Publications by an authorized administrator of DigitalCommons@University of Nebraska - Lincoln. 


\title{
Magnetic hardening in FePt nanostructured films
}

\author{
J. P. Liu, Y. Liu, C. P. Luo, Z. S. Shan, and D. J. Sellmyer \\ Behlen Laboratory of Physics and Center for Materials Research and Analysis, University of Nebraska, \\ Lincoln, Nebraska 68588-0113
}

FePt films have been prepared by sputtering Fe/Pt multilayers onto glass or silicon substrates. The thickness of the Fe and Pt layers was adjusted with the Fe:Pt atomic ratio from about 1:1 to 2:1. Magnetic hardening is observed after heat treatment at elevated temperatures, which led to coercivity values exceeding $20 \mathrm{kOe}$ in samples with an Fe:Pt ratio around 1.2:1. The hardening originates from the formation of the tetragonal FePt phase with high magnetocrystalline anisotropy and a favorable microstructure. Two-phase composite films containing hard and soft phases were obtained when the Fe:Pt ratio increased. Under optimized processing conditions, composite films with energy products larger than $30 \mathrm{MG}$ Oe at room temperature have been successfully produced.

(C) 1997 American Institute of Physics. [S0021-8979(97)21708-1]

\section{INTRODUCTION}

Recently, FePt thin films have drawn much attention for their potential application as high-density magnetic recording materials. ${ }^{1,2}$ One of the reasons for the great interest is the possibility of developing higher coercivity in the materials than that in the current recording media. The high coercivity in FePt comes from the large magnetocrystalline anisotropy of the ordered tetragonal $L 1_{0} \mathrm{FePt}$ phase and a favorable microstructure of the film. The anisotropy constant $K_{1}$ of FePt is $6.6 \times 10^{7} \mathrm{ergs} / \mathrm{cc}^{3}$, which is a value high enough for permanent magnetic materials. In view of this point, the FePt system is also an excellent candidate for permanent magnetic materials. Klemmer and co-workers have studied the magnetic hardening and coercivity mechanisms in the $L 1_{0}$ phases systematically. ${ }^{3}$ More than ten years ago, Watanabe and Msumoto ${ }^{4,5}$ studied the permanent magnetic properties of $\mathrm{Fe}-\mathrm{Pt}$ alloys. They obtained the highest coercivity $5.1 \mathrm{kOe}$ and maximum energy product $20 \mathrm{MG}$ Oe in the cast alloys. Yung et al. ${ }^{6}$ investigated the magnetic hardening of single-layer FePt thin films. They obtained a maximum coercivity of $13 \mathrm{kOe}$ and highest energy product $(B H)_{\max } 15$ MG Oe.

In this paper, we will focus on the magnetic hardening and the permanent magnetic properties of $\mathrm{Fe} / \mathrm{Pt}$ films.

\section{EXPERIMENT}

$\mathrm{Fe} / \mathrm{Pt}$ multilayer thin films were prepared with a multiple-gun dc- and rf-sputtering system by depositing $\mathrm{Fe}$ and Pt targets onto high-temperature operational glass or silicon substrates. The targets are commercial products with purities higher than 99.99 at. \%. The substrates were attached to a rotating table that was cooled by water during sputtering. The base pressure in the chamber was $2 \times 10^{-7}$ Torr. Flowing high-purity argon gas was used for sputtering and the pressures were varied from 5 to 20 mTorr. The thicknesses of the films were measured by weighing the mass of the films and by small-angle $x$-ray diffraction. The as-deposited films were then heat treated in a furnace with vacuum $2 \times 10^{-7}$ Torr and/or with rapid thermal processing equipment in which flowing high-purity argon gas was used to protect the samples from oxidation. The crystal structures of the films were identified using large-angle $\mathrm{x}$-ray diffraction with
$\mathrm{Cu} K_{\alpha}$ radiation. The microstructures of the films were observed by a JEOL 2010 transmission electron microscope (TEM). Magnetization loops were measured by a Micromag2900 alternating gradient force magnetometer and a quantum superconducting quantum interference device magnetometer.

The sputtering parameters in this investigation (including the argon pressure, the power rates of sputtering targets, and the distance between the targets and the substrates) have been chosen to obtain a deposition rate around $1 \AA / \mathrm{s}$ and to produce films with maximum coercivity. The thicknesses of the $\mathrm{Fe}$ and Pt layers in each period of the multilayer were adjusted according to the desired compositions.

\section{RESULTS AND DISCUSSION}

Figure 1 shows the small-angle $x$-ray diffraction pattern of a multilayer with a Fe thickness of $18 \AA$ and a Pt thickness of $15 \AA(\mathrm{Fe} 18 / \mathrm{Pt} 15)$ in each period. It is seen from Fig. 1 that the layered structure in the as-deposited film is evident and the bilayer thickness calculated from the pattern is $34 \AA$, which is in good agreement with the required value.

The as-deposited films behave like soft magnetic materials without significant coercivity, because iron is magnetically soft. High coercivity is developed after the samples are annealed in vacuum for half an hour. The coercivity increases with increasing annealing temperature. Figure 2

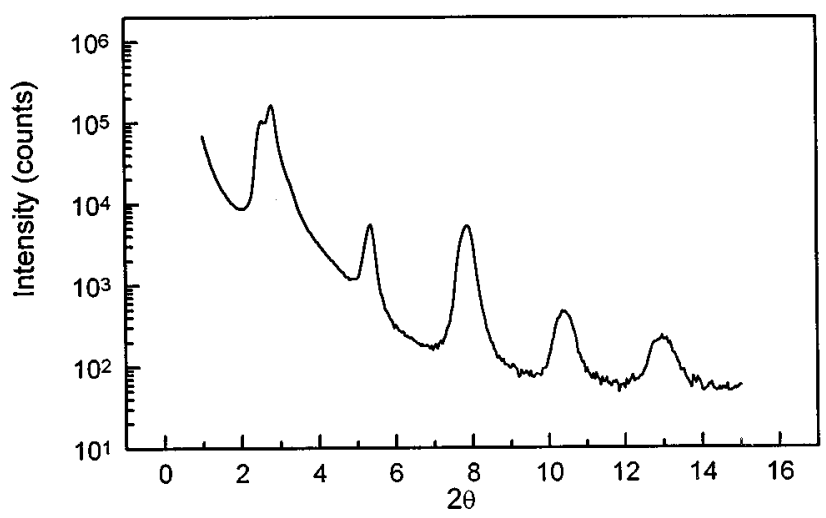

FIG. 1. Small-angle $\mathrm{x}$-ray diffraction pattern $(\mathrm{Cu} K \alpha$ radiation) of the $\mathrm{Fe} / \mathrm{Pt}$ multilayer film with structure $(\mathrm{Fe} 18 / \mathrm{Pt} 15) \times 8$. 


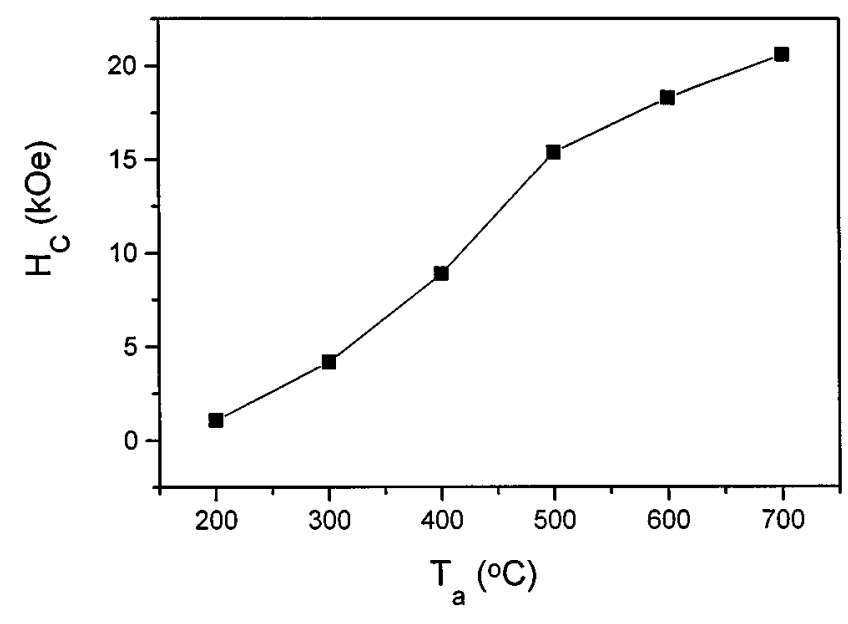

FIG. 2. Dependence of the coercive force of the $(\mathrm{Fe} 18 / \mathrm{Pt} 15) \times 8$ film on the annealing temperature, $T_{a}$. The lines are a guide to the eyes.

gives the dependence of the coercive force of the (Fe18/ Pt15) $\times 8$ film ( 8 is the number of the bilayers in the multilayer) on the annealing temperature. The coercive force was measured in the direction parallel to the film plane. When the annealing temperature exceeds $600{ }^{\circ} \mathrm{C}$, the anisotropy of the film changes from in plane to perpendicular, though the magnitude of the difference is not big. The details of the anisotropy of the films will be discussed elsewhere. ${ }^{7}$ In Fig. 3, the hysteresis loop of a sample with coercive force larger than $20 \mathrm{kOe}$ is demonstrated. This is the highest coercivity founded in the $\mathrm{Fe}-\mathrm{Pt}$ system to our knowledge.

Magnetic hardening of the annealed films is attributed to the formation of the high anisotropy FePt phase. Figure 4 is the $\mathrm{x}$-ray diffraction pattern of the sample after annealing at $700{ }^{\circ} \mathrm{C}$ for $30 \mathrm{~min}$. All the peaks can be identified as reflections from the ordered tetragonal FePt phase. The electron diffraction pattern confirms the presence of the tetragonal phase.

A favorable microstructure is also essential to high coercivity, because the change in domain structure and the motion of domain walls are highly sensitive to grain size and

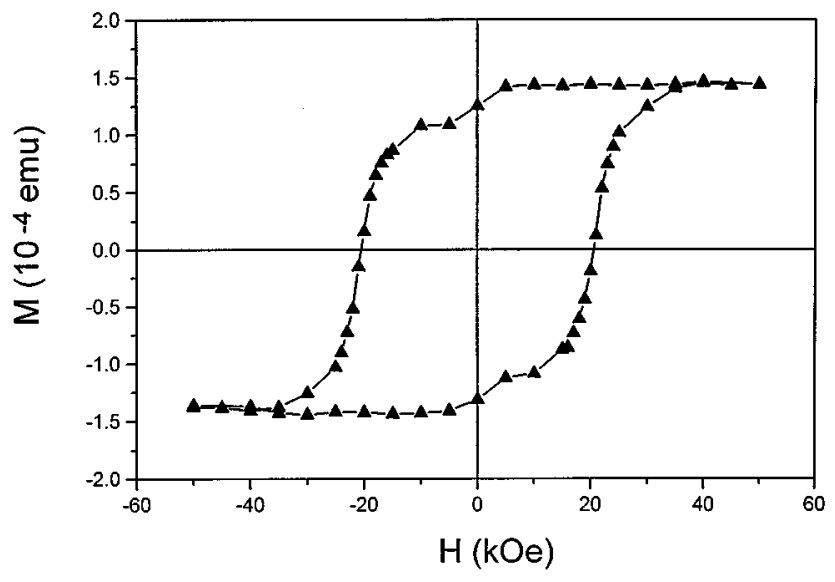

FIG. 3. Hysteresis loop measured along the plane of the $(\mathrm{Fe} 18 / \mathrm{Pt} 15) \times 8$ film annealed at $700{ }^{\circ} \mathrm{C}$ for $40 \mathrm{~min}$. The kink on the curve may be caused by the two-phase structure.

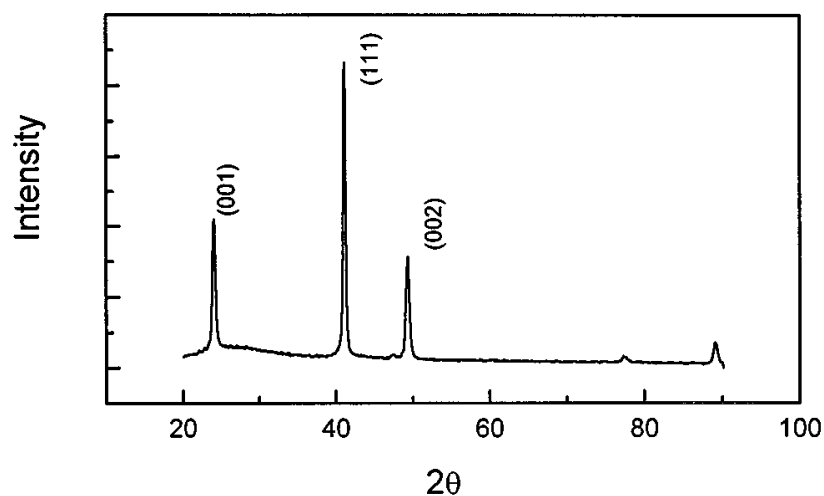

FIG. 4. Large-angle $\mathrm{x}$-ray diffraction patterns $(\mathrm{Cu} K \alpha$ radiation) of the $\mathrm{Fe} / \mathrm{Pt}$ films. (a) The as-deposited film and (b) the film after annealing at $700{ }^{\circ} \mathrm{C}$ for half an hour.

shape, the defect type and distribution, and the grain boundary structure. Figure 5 shows a TEM micrograph of a sample with coercivity larger than $20 \mathrm{kOe}$. It can be seen that the grain size is in the range from 50 to $100 \mathrm{~nm}$. Shorter annealing times produce smaller grain sizes. It has been found from our experiment that the coercivity is not very sensitive to the grain size in this range, suggesting that the pinning of domain walls by grain boundaries is not significant. Figure 5 also indicates that the film is basically a single phase. This can be attributed to the composition of the film (with period Fe18/Pt15), which is basically in the composition range of the FePt solid solution.

It is reasonable to assume that the grains of the $\mathrm{FePt}$ phase look like pies with their surface parallel to the surface of the films. This is not only because of the as-deposited structure of the films but also due to the large difference in the diffusion coefficients. The frequency factor $D_{0}$ for $\mathrm{Pt}$ diffusion in $\mathrm{Fe}$ is 100 times greater than that for $\mathrm{Fe}$ in $\mathrm{Pt},{ }^{8}$ making the interdiffusion almost in one way, from Pt layers to $\mathrm{Fe}$ layers. The formed grains preserve their twodimensional pielike shape. This microstructure causes some interesting phenomena in magnetization processes and the coercivity mechanism. We have found that if measured along the surface, the initial magnetization curve suggests a

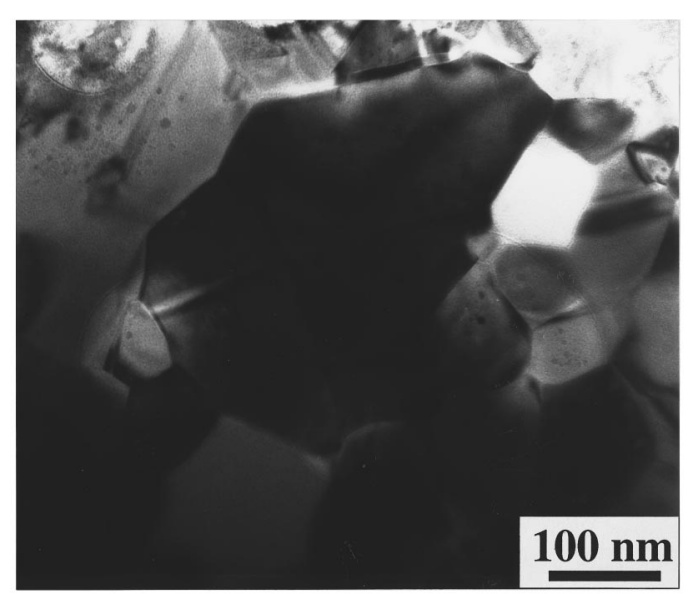

FIG. 5. TEM micrograph of the sample with hysteresis loop shown in Fig. 3. 
nucleation-controlled mechanism. If measured along the direction perpendicular to the surface, a typical wall-pinning mechanism is found.

Obtaining magnetic hardening is only the first step of our project. Our final goal in this research is to obtain films with high-energy products. Despite very high coercivity, the single-phase samples have relatively low saturation magnetizations and, therefore, low maximum energy products $(B H)_{\max }$. Especially for samples annealed for a long time at elevated temperature, the saturation magnetization is lower than $900 \mathrm{emu} / \mathrm{cc}$. (The saturation magnetization of the bulk alloy is higher than 1000 emu/cc; see Ref. 3.) This may be due to the fact that $\mathrm{Fe}-\mathrm{Pt}$ is a system in which there are a number of phases with complicated magnetic structures. For instance, the formation of some tiny grains of a phase with very low $M_{s}$ (like $\mathrm{Pt}_{3} \mathrm{Fe}$ ) will reduce the magnetization. Another possible reason is the increase of density upon the formation of the tetragonal phase compared with the asdeposited films. The oxidation-induced reduction in magnetization can also not be excluded for the samples annealed for more than half an hour at high temperatures.

A good way to increase the saturation magnetization is to increase the Fe:Pt ratio in the film; however, the coercivity decreases with increasing Fe concentration. Apparently, higher Fe content leads to the formation of a two-phase composite and it is very likely that the composite contains the FePt hard phase and free iron soft phase. Usually, the hysteresis loop of such a composite has two obvious kinks when the curve passes through the magnetization axis, suggesting a weak exchange coupling between the grains of the hard phase and the soft phase.

Calculations show that if the dimension of the soft phase is less than double the domain wall width in the hard phase, strong exchange coupling between the grains of these two phases can be realized and the ratio $M_{r} / M_{s}$ should be larger than 0.5. ${ }^{9}$ In the FePt tetragonal phase, the wall thickness is $3.9 \mathrm{~nm}^{3}$ This tells us that if the free iron grains in the $\mathrm{FePt} / \mathrm{Fe}$ composite are not bigger than $7.8 \mathrm{~nm}$, exchangecoupled magnets can be obtained. Fortunately, it is not dif- ficult to control the grain size in the sputtered films. The FePt hard phase can be formed in a very thin layer $(<2 \mathrm{~nm})$, making it possible to increase the fraction of the soft phase; however, the grains grow rapidly during the annealing. A method to control this growth is to use rapid thermal processing equipment, which allows annealing at temperatures higher than $500{ }^{\circ} \mathrm{C}$ for less than $5 \mathrm{~s}$. Careful thermal processing is needed to obtain a desired nanostructure. FePt/Fe films with energy products larger than 30 MGOe have been produced after a proper heat treatment. The details will be reported elsewhere. ${ }^{10}$

\section{CONCLUSION}

Magnetic hardening has been realized in sputtered Fe/Pt multilayer films after proper heat treatment. The highest coercivity exceeds $20 \mathrm{kOe}$. On this basis, exchange-coupled composite films with an energy product exceeding 30 MGOe have been produced.

\section{ACKNOWLEDGMENTS}

One of the authors (J.P.L.) is grateful to Professor S. Jaswal and Professor D. L. Leslie-Pelecky for fruitful discussions. This research was sponsored by the U.S. Department of Energy under Grant Nos. DOE-DE-F602-86ER45262 and DOE/ANL-95-47DH-007.

${ }^{1}$ C. P. Luo and D. J. Sellmyer, IEEE Trans. Magn. 31, 2764 (1995).

${ }^{2}$ C. P. Luo, Z. S. Shan, and D. J. Sellmyer, J. Appl. Phys. 79, 4899 (1996).

${ }^{3}$ T. Klemmer, D. Hoydick, H. Okumura, B. Zhang, and W. A. Soffa, Scr. Metall. Mater. 33, 1793 (1995).

${ }^{4}$ K. Watanabe and H. Msumoto, J. Jpn. Inst. Metals 48, 930 (1984).

${ }^{5}$ Kiyoshi Watanabe, J. Jpn. Inst. Metals 51, 91 (1987).

${ }^{6}$ S. W. Yung, Y. H. Chang, T. J. Lin, and M. P. Hung, J. Magn. Magn. Mater. 116, 411 (1992).

${ }^{7}$ J. P. Liu, Y. Liu, C. P. Luo, Z. S. Shan, and D. J. Sellmyer (unpublished).

${ }^{8}$ Diffusion in Solid Metals and Alloys, edited by H. Mehrer, LandoltBoernstein, New Series, group III (Springer, Berlin, 1990), Vol. 26.

${ }^{9}$ See, for example, R. Skomski and J. M. D. Coey, Phys. Rev. B 48, 15812 (1993).

${ }^{10}$ J. P. Liu, Y. Liu, C. P. Luo, Z. S. Shan, and D. J. Sellmyer (unpublished). 